\title{
QUANTITATIVE IMAGING OF MURINE OSTEOARTHRITIC CARTILAGE BY PHASE CONTRAST MICRO-COMPUTED TOMOGRAPHY
}

\author{
Merry Ruan ${ }^{1}$, Brian Dawson ${ }^{2}$, Ming-Ming Jiang ${ }^{2}$, Francis Gannon ${ }^{1}$, Michael Heggeness ${ }^{1}$, \\ and Brendan Lee ${ }^{1,2}$ \\ ${ }^{1}$ Baylor College of Medicine, Houston, TX, 77030 \\ ${ }^{2}$ Howard Hughes Medical Institute
}

\section{Abstract}

Objective-Mouse is an optimal model organism in which gene-environment interactions can be studied for the pathogenesis of osteoarthritis. The gold standard in arthritis research in mice is based on histopathology and immunohistochemistry, which are labor intensive, prone to sampling bias and technical variability, and limited in throughput. This study aims to develop a new technique that assesses mouse cartilage by integrating quantitative volumetric imaging techniques.

Methods-A novel mouse model of osteoarthritis was generated by cruciate ligament transection (CLT) and evaluated by histopathology and immunohistochemistry. Knee joint samples were then imaged by a novel technique that combines high resolution $\mu \mathrm{CT}$ and phase-contrast optics followed by quantitative analyses. A comparative analysis was also performed on a previously established mouse model of osteoarthritis generated by destabilization of medial meniscus (DMM).

Results-Phase contrast $\mu \mathrm{CT}$ achieved cellular resolution of chondrocytes and quantitative assessment of parameters such as articular cartilage volume and surface area. In mouse models of OA generated by either CLT or DMM, we showed that phase contrast $\mu$ CT distinguished control and OA cartilage by providing quantitative measures with high reproducibility and minimal variability. Features of $\mathrm{OA}$ at the cellular or tissue levels could also be observed in images generated by phase contrast $\mu \mathrm{CT}$.

Conclusion-We established an imaging technology that comprehensively assessed and quantified the 2 and 3 dimensional changes of articular cartilage. Application of this technology will facilitate the rapid and high throughput assessment of genetic and therapeutic models of OA in mice.

\section{Introduction}

Osteoarthritis (OA) is characterized by degeneration of articular cartilage, intra-articular inflammation with synovitis, and remodeling of peri-articular and subchondral bone. It is a major cause of disability and is one of the most common musculoskeletal disorders(1). The prevalence of the disease is also increasing as a consequence of demographic shifts and lifestyle(2). New treatment modalities are direly needed and animal models with reliable and informative endpoints have been a major focus of research efforts. Mouse models that mimic clinical OA are of importance because genetic analysis can be easily exploited, 
leading to development of new therapeutic modalities and pharmacological treatments. Like most other soft tissue diseases, the primary assessment of all existing mouse models of OA has been limited to standard histological scoring(3), which is time consuming and subject to artifact and inter-observer viability. It only provides semiquantitative analysis of 2D sections that may generate biased 3D representation.

High-resolution micro-computed tomography $(\mu \mathrm{CT})$ imaging has been established as the gold standard in assessing calcified tissue morphology such as trabecular and cortical bone in small animals at high resolution(4-7). However, this approach does not effectively image uncalcified soft tissues like cartilage. In situ imaging of soft tissue using equilibrium partitioning with an ionic contrast agent via micro-commutated topography (EPIC- $\mu \mathrm{CT}$ ) was shown to be successful in rabbits and rats using Hexabrix, an iodine-based staining reagent $(8,9)$. However, this procedure, when used in tissues other than blood vessels, required dissection with potential for introducing artifacts in small animals, and resolutions have been inadequate for imaging most soft tissue in mice.

To date, one of the most successful contrast stains used for $\mu \mathrm{CT}$ imaging of soft tissues and embryos has been osmium tetroxide $(10,11)$. In addition, Ruthenium hexamine trichloride (RHT) has yielded homogeneous, reproducible preservation of epiphyseal chondrocytes(12). To further increase the quality of cartilage imaging, we further applied phase contrast optical imaging. Phase-contrast imaging at optical wavelengths has been an important technique for thin biology samples without staining. Diffraction causes shifts in the relative phase of incident radiation (including X-ray) at the interfaces of tissues. In the context of imaging, they arrive at the detector out of phase with respect to others. Any detector sensitive to the direction of propagation of X-ray is therefore capable of detecting tissue surface even when the X-ray absorptions of the tissues are similar $(13,14)$. This provides the possibility of distinguishing tissue of interest from remaining soft tissues with similar absorbance.

Here, we present a new approach that combines contrast staining, phase contrast optics, high-resolution $\mu \mathrm{CT}$ and $3 \mathrm{D}$ graphical quantification. As an example, we investigated the visualization and quantification of articular cartilage and subchondral bone in a mouse model of osteoarthritis at cellular resolution. To generate a robust and reliable mouse model of osteoarthritis, we performed cruciate ligament transection of the mice with minimal injury to surrounding tissue. OA generated by this procedure mimics post-traumatic OA in human patients, as ligament tear is a common cause of OA. We assessed our model by classical histology and immunohistochemistry. After validating our mouse model using established technology, we scanned the knee joints in situ and analyzed the samples in three different ways. First, we observed the samples in 2D for pathological changes. Second, we reconstructed the joints in 3D and observed OA traits comprehensively. This provided direct visualization of cartilage degeneration and bone remodeling. Last, to further enhance the reliability and objectiveness of the method, we developed algorithms that facilitated the quantification of volume and area measurements based on the 3-dimensional (3D) platform. Since the destabilization of medial meniscus (DMM) in a genetically modified mouse is commonly used in evaluating the impact of therapeutic modifications in $\mathrm{OA}(15,16)$, we compared our analytic approach and our mouse model of OA to the DMM model using histology scores and quantification of cartilage volume and area. This technology provides a novel and robust endpoint for assessment of OA, and potentially other soft tissue diseases with high throughput. 


\section{Material and Methods}

\section{Animals}

FVB/N and 129SV/EV mice were purchased from Jackson Laboratories (Bar Harbor, ME). This strain was used since this is a common background strain for transgenic mouse lines. All studies were performed with approval from the Baylor College of Medicine Institutional Animal Care and Use Committee (IACUC). All mice were housed under pathogen-free conditions in groups of four to five per cage. Mice had free access to feed and water. To avoid potential post-menopausal bone loss, all the mice used in the experiments were males.

\section{Cruciate ligament transection surgery}

8-week old male FVB/N mice were anesthetized by intraperitoneal injection of $0.2 \mathrm{ml} / 10$ grams $1.2 \%$ tribromoethanol (Sigma-Aldrich) and knees were prepared for aseptic surgery. Appropriate skin preparation for a sterile procedure consisted of hair removal, cleansing with surgical soap followed by with $70 \%$ alcohol wash. The cleaning procedure was repeated for three times. To provide better view of the knee joint space, No.11 feather disposable scalpel (Feather safety razor Co., Ltd) was used to provide a skin-deep $\sim 5 \mathrm{~mm}$ incision in the medial side of the joint parallel to the patellar tendon. Bvi Beaver EdgeAhead Safety knife Sideport MVR $0.90 \mathrm{~mm} 20 \mathrm{G}$ microsurgical scalpel (Beaver-Visitec International, Inc.) was inserted into the knee joint space from the side of the patellar tendon. The transection of the anterior and posterior cruciate ligament was made in the CLT group; and no transection was made in the sham group. The success of the transection could be tested by valgus and varus laxity of the knee. The surgery was associated with no blood loss when properly performed. The skin was closed by application of 5.0 monofilament sutures (Ethicon Inc.). Sutures were removed 2 weeks after surgery.

\section{Destabilization of medial meniscus}

The model was generated via standard approaches that have been previously described(16).

\section{Histology and immunohistochemistry}

Mice were euthanized and whole knee joints were fixed with 4\% paraformaldehyde (SigmaAldrich) overnight in $4^{\circ} \mathrm{C}$ on a shaker. Whole knee joints were decalcified in $14 \%$ EDTA for 5 days in $4^{\circ} \mathrm{C}$ on a shaker. After dehydration by gradient alcohol and infiltration by xylene and paraffin, samples were embedded in paraffin. Paraffin-embedded joints were sectioned at $6 \mu \mathrm{m}$ on a sagittal plane. Samples were stained with safranin $O$ and fast green using standard protocols. Immunohistochemistry were performed using primary antibody: rabbit anti mouse ColX (generous gift from Dr. Greg Lunstrum, Shriners Hospital for Children, Portland, OR) and mouse anti-human MMP13 monoclonal antibody (Millipore), and secondary antibody: one-dropper-bottle HRP polymer conjugates (Invitrogen). Histomark trueblue (KPL) was used as developing reagent. Samples without primary antibody treatment served as negative controls.

\section{Phase contrast $\mu \mathrm{CT}$ scanning}

Whole joint knees were dissected and fixed with ruthenium hexamine tricholoride $(0.7 \%$, Sigma-Aldrich), glutaraldehyde ( $0.2 \% \mathrm{v} / \mathrm{v}$, Polysciences, Inc.), cacodylic acid $(0.05 \mathrm{M}$, Sigma-Aldrich), $\mathrm{pH} 7.4$ for 24 hours to allow for its complete penetration. Afterwards, the samples were washed $3 \times 30$ minutes with ruthenium hexamine tricholoride $(0.7 \%)$, cacodylic acid $(0.05 \mathrm{M}), \mathrm{pH} 7.4$. Samples were then fixed for 24 hours with ruthenium hexamine tricholoride $(0.7 \%)$, osmium tetroxide ( $1 \%$, Polysciences, Inc), cacodylic acid $(0.1 \mathrm{M}), \mathrm{pH}$ 7.4. After dehydration by gradient alcohol and infiltration by xylene, samples were embedded in paraffin. Before scanning, excess paraffin was removed and the samples were 
mounted and stabilized on an Xradia sample holder. Samples were then scanned by Xradia $\mu \mathrm{XCT}$ with conditions listed in table 1 .

\section{Reconstruction and analysis of $\mu \mathrm{CT}$ data}

Reconstruction of the data was performed using Xradia software and was transformed into dicom files. Reconstruction involves correction for beam hardening, and correcting for center shift effects caused by difference between the center of sample rotation and the center of the detector. Samples were analyzed using TriBON software (RATOC, Tokyo, Japan).

\section{Statistics}

Statistical significance comparing two groups with parametric data was assessed by Student's $t$ test. Statistical analysis comparing multiple groups with parametric data was performed by one-way ANOVA followed by Tukey's post-hoc. Rotarod analysis was tested by one-way ANOVA with repeated measures followed by Tukey's post-hoc. Histological grades were compared by Wilcox rank test. All analyses were performed by SPSS software. A P value of $<0.05$ was considered statistically significant.

\section{Results}

\section{Mouse Model of OA by Cruciate Ligament Transection: Histological and functional Outcome}

Anterior cruciate ligament (ACL) and posterior cruciate ligament (PCL) injury are common causes of OA in humans $(17,18)$ ENREF_6. To generate an OA mouse model that mimics this clinical condition, anterior and posterior cruciate ligament transections (CLT) were performed in the mouse via a microsurgical approach while leaving the patellar tendon intact. Laxity of the joint in response to valgus and varus stress confirmed the transection of ligaments. In the sham surgery, a similar invasive procedure was conducted, but without actual ligament transection (Fig. 1A). Continuous histological sections collected 24 hours after transection confirmed that the patella was intact and that no visible damage was evident on either the joint surface or the meniscus in both the ligament transected group or the sham surgery group. To assess the severity of OA generated by our technique microscopically, histological grading was performed by two independent masked observers using standard OARSI scores(3). The sham operated limb demonstrated insignificant levels of OA whereas histologically significant OA was observed in the limbs with CLT over time (Fig. 1B, C). In addition, accelerated cartilage hypertrophy was observed in OA joints, as demonstrated by increased immunohistochemical staining using anti-MMP13 and anti-ColX antibodies (Fig. 1C). These data show that this surgical model produced the pathognomonic features of OA on standardized histological assessment and may serve as a clinically relevant OA model.

\section{Morphological evidence of OA assessed by phase contrast $\mu \mathrm{CT}$}

Phase contrast $\mu \mathrm{CT}$ was utilized and a scanning protocol was established to image cartilage at ultrahigh resolution. Intact knees were fixed and infiltrated with Ruthenium hexamine trichloride (RHT), a reagent that increases contrast in soft tissue and preserves chondrocyte morphology, and osmium tetroxide to create high contrast in cell membrane $(12,19)$. Samples were embedded in paraffin and later scanned in situ to visualize all tissues.

Scanning of the OA joints was performed at different resolutions. At $10 \mu \mathrm{m}$ resolution, an overview of the entire joint showed high-density materials including meniscus and bone. At the $4 \mu \mathrm{m}$ resolution, the cartilaginous area in the joint could be imaged. At the $2.03 \mu \mathrm{m}$ resolution, a single mouse knee joint condyle was shown, providing soft tissue visualization and cellular detail. At the $1.54 \mu \mathrm{m}$ and $0.54 \mu \mathrm{m}$ resolutions, a limited area of joints could be 
pictured to show changes in cell distribution as demonstrated by cell lacunae (Fig. 2A). At the tissue level, OA joints displayed loss of uncalcified cartilage. And at the cellular level, OA joints showed chondrocytes clustering while sham joints showed evenly distributed chondrocytes (Fig. 2A). After comparing different resolutions (Table 1), we concluded that cartilage could be clearly imaged at 4 and $2.03 \mu \mathrm{m}$ scanning resolution. In the interest of throughput, all joints were scanned at $4 \mu \mathrm{m}$ in subsequent analyses to assess the morphological signs and symptoms associated with OA progression (Fig. 2A).

Articular cartilage loss due to mechanical stresses is the first step in osteoarthritis. Once articular cartilage completely disappears, bone on bone contact in the joint causes pain. Loss of cartilage triggers cellular proliferation that results in hypertrophic bone remodeling. Phase contrast $\mu \mathrm{CT}$ was capable of visualizing cartilage loss (Fig. 2B). In some osteoarthritic knees, we also observed free floating cartilage fragments (Fig. 2C). Meniscus metaplasia transforms meniscus from a cartilaginous tissue to an osseous tissue. This process causes uneven weight distribution in the knee and excessive forces in specific areas, leading to more localized articular cartilage loss. In almost all knee joints in the CLT group, at least one meniscus underwent changes in size, cellular architecture, and density. Histological observations corroborated the phase contrast $\mu \mathrm{CT}$ findings (Fig. 2D). Chondrocyte hypertrophy above the tide mark best shown by increased matrix metallopeptidase 13 (MMP13) and Collagen type X (ColX) expression is a biomarker of altered cartilage and bone remodeling in OA (Fig. 1C). It leads to subchondral bone remodeling, which is characterized by erosion, denudation, and in severe cases, micro-fracture. These signs can occur only regionally within the knee joint and can be easily missed by histological sampling. For example, erosion and denudation were visualized especially in the edge of cartilage by analysis of the whole joint using phase contrast $\mu \mathrm{CT}$ (Fig. 2E). Micro-fractures, even in cases that were not easily identified by histological analysis, were detected in multiple sites by scanning through OA knee joints (Fig. 2F, G). Osteophyte formation near the articular surface (Fig. 2E) and heterotopic ossification in patellar tendons (Fig. 2H) were also observed in severe cases of OA.

\section{D reconstruction of the OA knee joint}

To assess cartilage in volumetric fashion, 3D reconstruction of the $\mathrm{CT}$ images was performed. After 3D reconstruction, data analysis was performed using RATOC TriBON software. High density regions (bone and fixative saturated lipid rich tissues) were assigned a mask and a hole filling algorithm was used to include marrow space with surrounding cortical and trabecular bone (mask1). Due to the similarities in density of the cartilage and surrounding soft tissue, the joint surfaces of femoral and tibial cartilage were defined manually and each assigned a mask (mask 2 and 3, respectively). Cartilage volume was defined by subtracting mask 1 from mask 2/3, resulting in mask 4 (femoral cartilage volume, labeled in red) and 5 (tibial cartilage volume, labeled in green) respectively (Fig. $3 \mathrm{~A}-\mathrm{H}$ ). The 3D reconstructed joint revealed cartilage volume loss, meniscus metaplasia, bone remodeling and osteophyte formation in CLT joints (Video S1). The joint was also imaged in various planes, such as coronal and sagittal, commonly used in the scoring of OA (Video S2).

\section{Quantification of OA cartilage volume and surfaces}

To objectively assess changes in articular cartilage and subchondral bone in a quantitative manner, volumes of masks 4 and 5 were calculated. Significant decreases in the tibial and total articular cartilage volumes were observed 1 and 2 months after CLT. Significant loss of femoral articular cartilage volume was detected only after 2 months, suggesting that the loss of tibial articular cartilage was a more prominent feature in this OA model. Three months after surgery, all CLT mice showed significant loss of cartilage at all condyles (Fig. 4A). 
Cartilage surface area changes may also reflect cartilage loss. In order to calculate cartilage surface area, mask 4/5 was 2D dilated and subtracted from mask 4/5 and mask 1, leaving only the joint cartilage surface. One month after surgery, no significant surface area loss was observed except in the lateral femoral condyle, which showed a statistically significant difference in the CLT mice vs. sham surgery mice (Fig. 4B). This piece of data, together with the quantification of cartilage volume, shows that that during the first month of OA development, the cartilage became thinner, but subchondral bone coverage was not lost. Two months after surgery, there was significant loss in the femoral, tibial, and total articular cartilage volumes (Fig. 4B). Three months after surgery, all condyles had significant loss of cartilage surface area, showing a progression of cartilage surface loss in this OA surgical mouse model (Fig. 4B).

To further quantify the progression of uncovered subchondral bone, bone surface area that was covered by cartilage in OA joints was calculated. This was assigned as the common volume of mask 1 and the dilated region of mask 4/5. One month and two months after surgery, we observed a significant decrease in most bone surface areas covered by cartilage (Fig. 4C). Three months after surgery, all condyles showed a significant increase in boneexposed surface (Fig. 4C).

Osteophyte formation and heterotopic bone formation are hallmarks of late stage OA, and a pathological sign of bone remodeling. Osteophyte and heterotopic bone numbers and volume were quantified by 3D labeling objects larger than 5000 voxels from mask 1 (bone and high density objects). Volumes were deselected for femur, tibia, fibula and menisci. Remaining volumes were assigned as osteophytes. No osteophytes or heterotopic bone formation were detected in the control knee joints. Both the number and volume of osteophytes increase with time after surgery in CLT group (Fig. 4D, E). Most joints in the CLT group developed osteophytes approximately 2 months after surgery. Three months after surgery, mice presented with a large number of osteophytes, suggesting severe remodeling of bone and soft tissue and late stage OA.

\section{Comparison between CLT and the previously established DMM model}

Destabilization of medial meniscus model of osteoarthritis is considered a mild model of osteoarthritis. It has been used to investigate genetic contribution of OA and treatment approaches to osteoarthritis. To compare the different features of OA induction by DMM vs. CLT and effects of different mouse strains on OA development, we compared our CLT model with DMM model generated in both FVB/N strain and 129SV/EV strains. The 129 $\mathrm{SV} / \mathrm{EV}$ is a commonly used strain in generating knockout mouse models and was the first stain on which DMM was performed. One month after DMM, FVB/N mice developed slightly less severe OA compared to 129SV/EV when evaluated by OARSI scoring (Fig. 5A, $E$ and data not shown). In addition, when evaluated by phase contrast $\mu \mathrm{CT}$ quantification, mice with DMM surgery showed loss of medial cartilage volume and area (Fig. 5B-D, FG). Interestingly, DMM also caused loss of lateral femoral cartilage volume and area, while no significant loss of lateral tibial cartilage was observed (Fig. 5B-D, F-G). This might be caused by the differential thickness and shape between femoral and tibial cartilage. It underscores that OA changes in the medial compartment does affect lateral articular cartilage volumes. No osteophytes formation was detected in the DMM model (data not shown).

After DMM, FVB/N seem to develop OA slower (Fig. 5B-D, F-G) than in 129SV/EV. This is probably due to size and activity differences between the two strains. Maximum histology scores of medial condyle in DMM joints were comparable to the maximum histology scores in CLT joints at one month after surgery (Fig. 1B, Fig. 5A, E). Interestingly, cartilage surface area losses in DMM joints were more severe than in those in CLT joints (Fig. 4B, 
5G). The variability (as assessed by standard deviation) of both the histology scores and

$\mu \mathrm{CT}$ quantification of DMM joints were slightly smaller than those of CLT joints,

suggesting DMM might be a more consistent method (Fig. 1B, 4A-C, 5).

\section{Discussion}

These results establish that phase contrast $\mu \mathrm{CT}$ is capable of visualizing signs of $\mathrm{OA}$ such as articular cartilage loss, meniscus metaplasia, subchondral bone remodeling, and osteophyte formation. It is also the first time that changes in OA knee joints in mice have been assessed quantitatively using a 3D imaging approach. The changes of OA knee joints in mice observed by phase contrast $\mu \mathrm{CT}$ correlate well with traditional methods such as histological grading and functional studies. Because phase contrast $\mu \mathrm{CT}$ data are available in all possible sectioning planes and are acquired from intact specimens with the potential for cellular resolution, the approach affords the power of cellular analysis in histology and whole joint analysis in functional studies, but with higher fidelity, higher throughput, and lower variability in quantitative fashion. When this technique was used to evaluate OA model by DMM, histology scoring could not detect statistically significant changes in the lateral condyle of the DMM joint while phase contrast $\mu \mathrm{CT}$ detected femoral cartilage loss. This supports that phase contrast $\mu \mathrm{CT}$ is a more sensitive method for detection of disease progression. At the same time, subchrondral bone could be easily assessed. On the basis of these features of phase contrast $\mu \mathrm{CT}$ analysis, we believe that it will have substantial impact on dissecting OA pathogenesis and developing treatment by bringing to bear the significant advantages of mouse models including genetic manipulation and lower cost.

Compared to traditional standard resolution $\mu \mathrm{CT}$, which is the standard for imaging mineralized tissues from mice $(20,21)$, phase contrast $\mu \mathrm{CT}$ protocols require optimization of tissue processing and data acquisition for different soft tissues. Different analysis protocols and landmarks will still need to be defined when analyzing new types of tissues. Until now, when applied in low resolution mode without tissue staining, phase contrast $\mu \mathrm{CT}$ has served much like standard $\mu \mathrm{CT}$ for evaluating bone remodeling in joint diseases(7). When combined with ionized contrast staining such as Hexabrix(8), phase contrast $\mu \mathrm{CT}$ may provide sufficient resolution to assess proteoglycan loss in cartilage in mouse OA models, which would be a future application for this approach in the context of OA modeling. With other contrast reagents such as Microfil $(22,23)$, it may also allow imaging of neovascularization.

Compared to micro magnetic resonance imaging ( $\mu$-MRI), which is widely used in soft tissue imaging, phase contrast $\mu \mathrm{CT}$ still does not show soft tissue boundaries as effectively $(24,25)$. However, this problem may be solved by investigating new contrast staining reagents that mark the tissue of interest, such as phosphotungstic acid for lysine and arginine residues in cartilage matrix(26). Scanning in different mediums such as air or agarose may also increase soft tissue contrast as well as expedite tissue processing. Importantly, phase contrast $\mu \mathrm{CT}$ provides imaging resolution that is an order of magnitude higher than $\mu$-MRI. This provides opportunities to quantify soft tissue volume and surface area in small animals, opening avenues for studying developmental phenotypes using mouse models. Moreover, the samples for phase contrast $\mu \mathrm{CT}$ can be preserved long term, allowing reanalysis if necessary. Finally, the shorter scanning time of phase contrast $\mu \mathrm{CT}$ allows higher analysis throughput than $\mu \mathrm{MRI}$.

Our understanding of OA is based largely on histological and immunohistochemical studies. A more comprehensive and quantitative imaging modality, such as phase contrast $\mu \mathrm{CT}$ that allows observation of cellular features of OA in the context of the whole knee joint, may shed new light on the pathogenesis of OA. 


\section{Supplementary Material}

Refer to Web version on PubMed Central for supplementary material.

\section{Acknowledgments}

We thank Terry Bertin and Ayelet Erez for suggestions and comments on the manuscript; Corinne Spencer for training in functional analysis; Greg Lunstrum (Shriners Hospital for Children) for collagen type X antibody; Stephen Henry (MD Anderson Cancer Center) for suggestions in immunohistochemistry. This work was supported by the Rolanette and Berdon Lawrence Bone Disease Program of Texas.

\section{References}

1. Schwartz ST, Zimmermann B. Update on osteoarthritis. Med Health R I. 1999; 82(9):321-4. [PubMed: 10517076]

2. Mollenhauer JA, Erdmann S. Introduction: molecular and biomechanical basis of osteoarthritis. Cell Mol Life Sci. 2002; 59(1):3-4. [PubMed: 11846030]

3. Glasson SS, Chambers MG, van den Berg WB, Little CB. The OARSI histopathology initiative recommendations for histological assessments of osteoarthritis in the mouse. Osteoarthritis and cxartilage / OARSI, Osteoarthritis Research Society. 2010; 18(S3):S17-S23.

4. Bouxsein ML, Boyd SK, Christiansen BA, Guldberg RE, Jepsen KJ, Müller R. Guidelines for assessment of bone microstructure in rodents using micro-computed tomography. J Bone Miner Res. 2010; 25(7):1468-86. [PubMed: 20533309]

5. Jiang Y, Zhao J, Geusens P, Liao EY, Adriaensens P, Gelan J, et al. Femoral neck trabecular microstructure in ovariectomized ewes treated with calcitonin: MRI microscopic evaluation. J Bone Miner Res. 2005; 20(1):125-30. [PubMed: 15619678]

6. Rupprecht M, Pogoda P, Mumme M, Rueger JM, Puschel K, Amling M. Bone microarchitecture of the calcaneus and its changes in aging: a histomorphometric analysis of 60 human specimens. $J$ Orthop Res. 2006; 24(4):664-74. [PubMed: 16514636]

7. Lee YS, Heo E-A, Jun HY, Kang SH, Kim HS, Lee MS, et al. Articular Cartilage Imaging by the Use of Phase-Contrast Tomography in a Collagen-Induced Arthritis Mouse Model. Academic Radiology. 2010; 17(2):244-50. [PubMed: 19962916]

8. Palmer AW, Guldberg RE, Levenston ME. Analysis of cartilage matrix fixed charge density and three-dimensional morphology via contrast-enhanced microcomputed tomography. Proc Natl Acad Sci U S A. 2006; 103(51):19255-60. [PubMed: 17158799]

9. Xie L, Lin ASP, Levenston ME, Guldberg RE. Quantitative assessment of articular cartilage morphology via EPIC-microCT. Osteoarthr Cartil. 2009; 17(3):313-20. [PubMed: 18789727]

10. Johnson JT, Hansen MS, Wu I, Healy LJ, Johnson CR, Jones GM, et al. Virtual histology of transgenic mouse embryos for high-throughput phenotyping. PLoS Genet. 2006; 2(4):e61. [PubMed: 16683035]

11. Bentley MD, Jorgensen SM, Lerman LO, Ritman EL, Romero JC. Visualization of threedimensional nephron structure with microcomputed tomography. Anat Rec (Hoboken). 2007; 290(3):277-83. [PubMed: 17525936]

12. Hunziker EB, Herrmann W, Schenk RK. Ruthenium hexammine trichloride (RHT)-mediated interaction between plasmalemmal components and pericellular matrix proteoglycans is responsible for the preservation of chondrocytic plasma membranes in situ during cartilage fixation. J Histochem Cytochem. 1983; 31(6):717-27. [PubMed: 6341460]

13. Davis T, Gao D, Gureyev T, Stevenson A. Phase-contrast imaging of weakly absorbing materials using hard X-rays. Nature. 1995

14. Wilkins S, Gureyev T, Gao D, Pogany A. Phase-contrast imaging using polychromatic hard Xrays. Nature. 1996

15. Glasson SS, Askew R, Sheppard B, Carito B, Blanchet T, Ma H-L, et al. Deletion of active ADAMTS5 prevents cartilage degradation in a murine model of osteoarthritis. Nature. 2005; 434(7033):644-8. [PubMed: 15800624] 
16. Glasson SS, Blanchet TJ, Morris EA. The surgical destabilization of the medial meniscus (DMM) model of osteoarthritis in the 129/SvEv mouse. Osteoarthr Cartil. 2007; 15(9):1061-9. [PubMed: 17470400]

17. Goldring MB, Goldring SR. Osteoarthritis. Journal of Cellular Physiology. 2007; 213(3):626-34. [PubMed: 17786965]

18. Haq I, Murphy E, Dacre J. Osteoarthritis. Postgrad Med J. 2003; 79(933):377-83. [PubMed: 12897215]

19. Stefanini M, De Martino C, Zamboni L. Fixation of ejaculated spermatozoa for electron microscopy. Nature. 1967; 216(5111):173-4. [PubMed: 4862079]

20. Rüegsegger P, Koller B, Müller R. A microtomographic system for the nondestructive evaluation of bone architecture. Calcified tissue international. 1996; 58(1):24-9. [PubMed: 8825235]

21. Jiang Y, Zhao J, Liao E-Y, Dai R-C, Wu X-P, Genant HK. Application of micro-CT assessment of $3-\mathrm{D}$ bone microstructure in preclinical and clinical studies. Journal of bone and mineral metabolism. 2005; 23 (Suppl):122-31. [PubMed: 15984427]

22. Carey DJ. Syndecans: multifunctional cell-surface co-receptors. The Biochemical journal. 1997; 327 ( Pt 1):1-16. [PubMed: 9355727]

23. Factor SM, Okun EM, Minase T. Capillary microaneurysms in the human diabetic heart. The New England journal of medicine. 1980; 302(7):384-8. [PubMed: 7351930]

24. Hipp JA, Jansujwicz A, Simmons CA, Snyder BD. Trabecular bone morphology from micromagnetic resonance imaging. Journal of bone and mineral research : the official journal of the American Society for Bone and Mineral Research. 1996; 11(2):286-97. [PubMed: 8822353]

25. Henkelman RM, Stanisz GJ, Graham SJ. Magnetization transfer in MRI: a review. NMR in Biomedicine. 2001; 14(2):57-64. [PubMed: 11320533]

26. Van Slyke DD. The analysis of proteins by determination of the chemical groups characteristic of the different amino-acids. The Journal of biological chemistry. 1911; 10(1):15. 
A

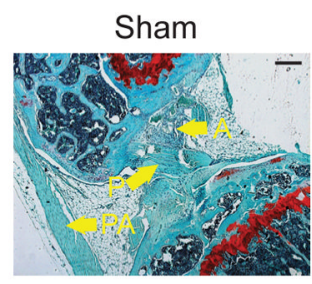

CLT
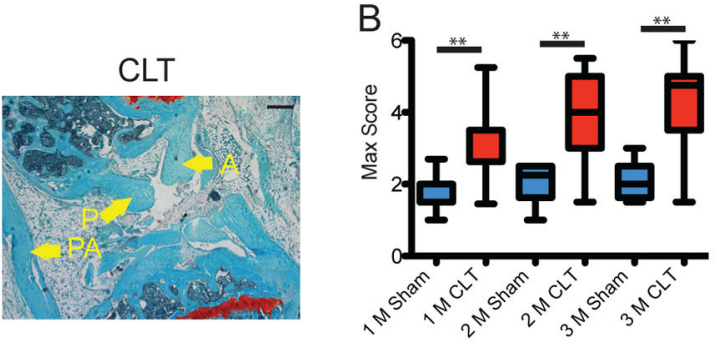

C

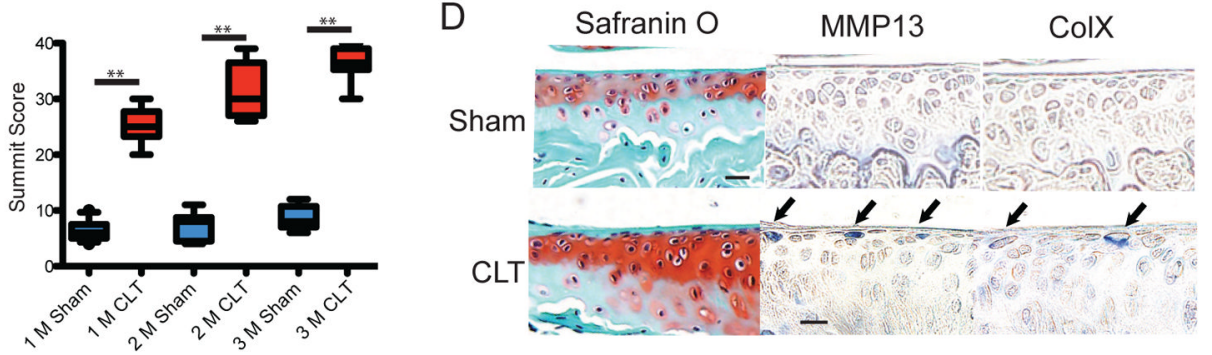

Figure 1. Histological characterization of the cruciate ligament transection model

(A) Safranin O staining of intercondylar fossa of mouse hind limb in sham (left) and CLT surgery (right). Anterior cruciate ligament, posterior cruciate ligament and patellar tendon are labeled as A, P and PA. Scale bar, 200 $\mu \mathrm{m}$. (B,C) Box plots of histology maximum score (B) and summed score (C) of cruciate ligament transected knees (CLT) compared to sham transection (sham) at 1 month, 2 months and 3 months after surgery ( $\mathrm{n}=8$, box plot showing median and interquartile range, whiskers represent 10 to 90 percentile). (D) Sham and cruciate ligament transected (CLT) joints stained with safranin O and anti- MMP13 and antiColX antibodies. Arrows indicate positive cells above tide marks. Scale bar, 50 $\mu \mathrm{m}$. All the significant comparisons are labeled, ${ }^{*} \mathrm{p}<0.05,{ }^{* *} \mathrm{p}<0.01$. 


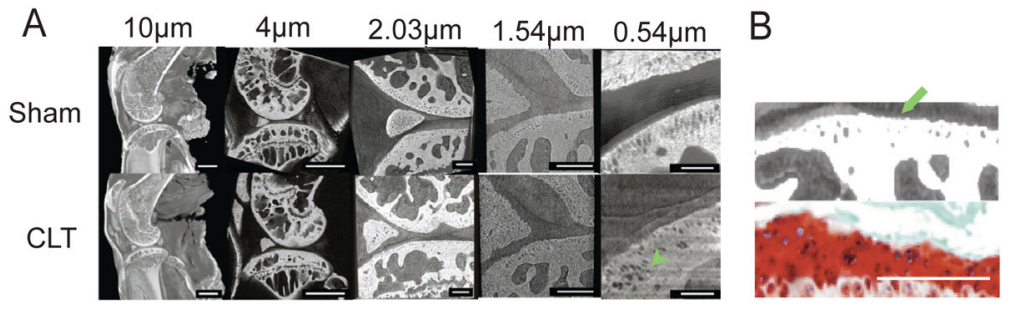

C
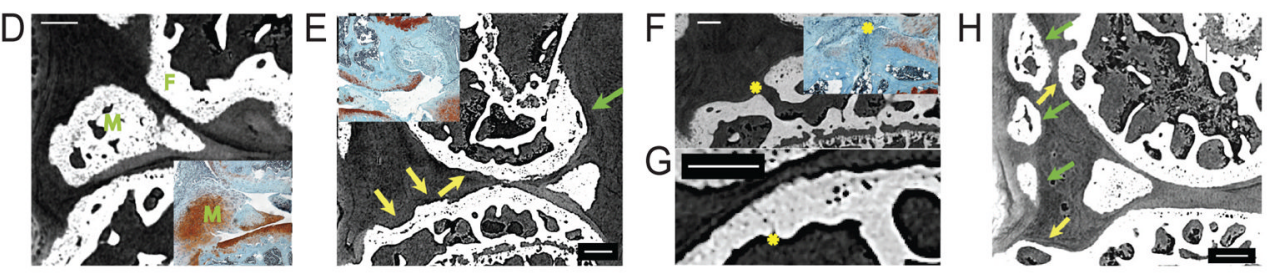

Figure 2. Phase contrast $\mu \mathrm{CT}$ images of sham and CLT joints

(A) Images of sham transected and CLT joints at different resolutions in phase contrast $\mu C T$. Scale bars, $1 \mathrm{~mm}$ in $10 \mu \mathrm{m}$ and $4 \mu \mathrm{m}$ resolutions; scale bars, $200 \mu \mathrm{m}$ in $2.03 \mu \mathrm{m}$ and $1.54 \mu \mathrm{m}$ resolutions; scale bars, $100 \mu \mathrm{m}$ in $0.54 \mu \mathrm{m}$ resolution, green arrow indicate cell clustering. (B-G) Features of OA represented by phase contrast $\mu \mathrm{CT}$ with comparison to safranin $\mathrm{O}$ staining. (B) Loss of cartilage, (C) cartilage proliferation, (D) meniscus metaplasia, femur is labeled as F, and meniscus labeled as M. (E) Erosion (green arrow) and bone remodeling (yellow arrows) in comparison with erosion shown on slides stained with safranin O. (F, G) Micro-fractures indicated by a yellow asterisk. (H) Osteophyte formation (yellow arrows) and sesamoid bone in patellar tendon (green arrows). Scale bars, $200 \mu \mathrm{m}$. 

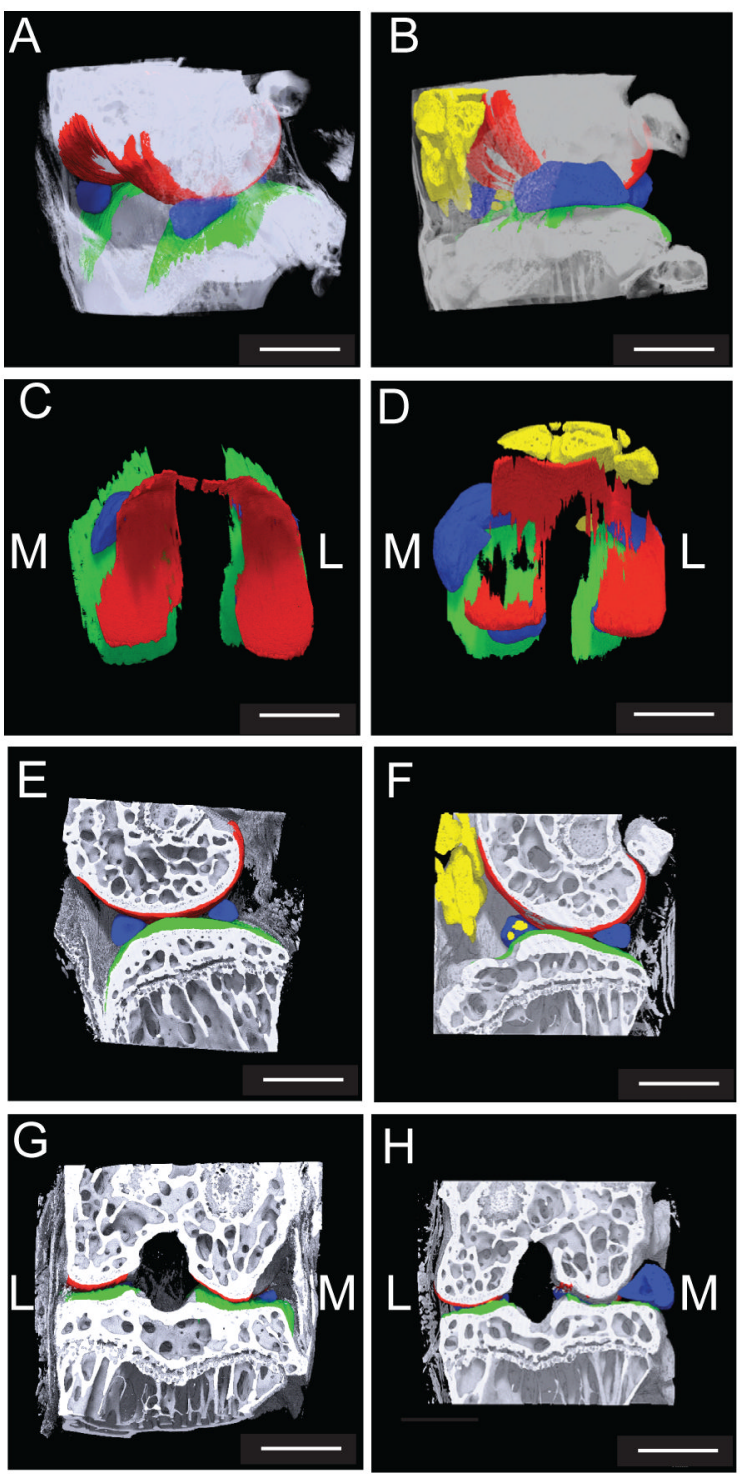

Figure 3. Phase contrast $\mu \mathrm{CT}$ images of sham transected and CLT joints

(A-D) Static picture of 3D reconstructed joint in a $4 \mu \mathrm{m}$ scan. (E-H) Sagittal sections (E, F) and coronal sections $(\mathbf{G}, \mathbf{H})$ of knee joints from phase contrast $\mu \mathrm{CT}$. (A, C, E, G)

Representative knee joint 3 months after sham surgery ( 6 joints in each group). (B, D, F, H) Representative knee joint 3 months after CLT ( 6 joints in each group). Bone is shown in white, femoral and tibial cartilage in red and green, respectively, menisci in blue, and osteophytes and patella metaplasia in yellow. In (A) and (B), the closer condyle is the medial condyle. M: medial, L: lateral. Scale bars, $1 \mathrm{~mm}$. 

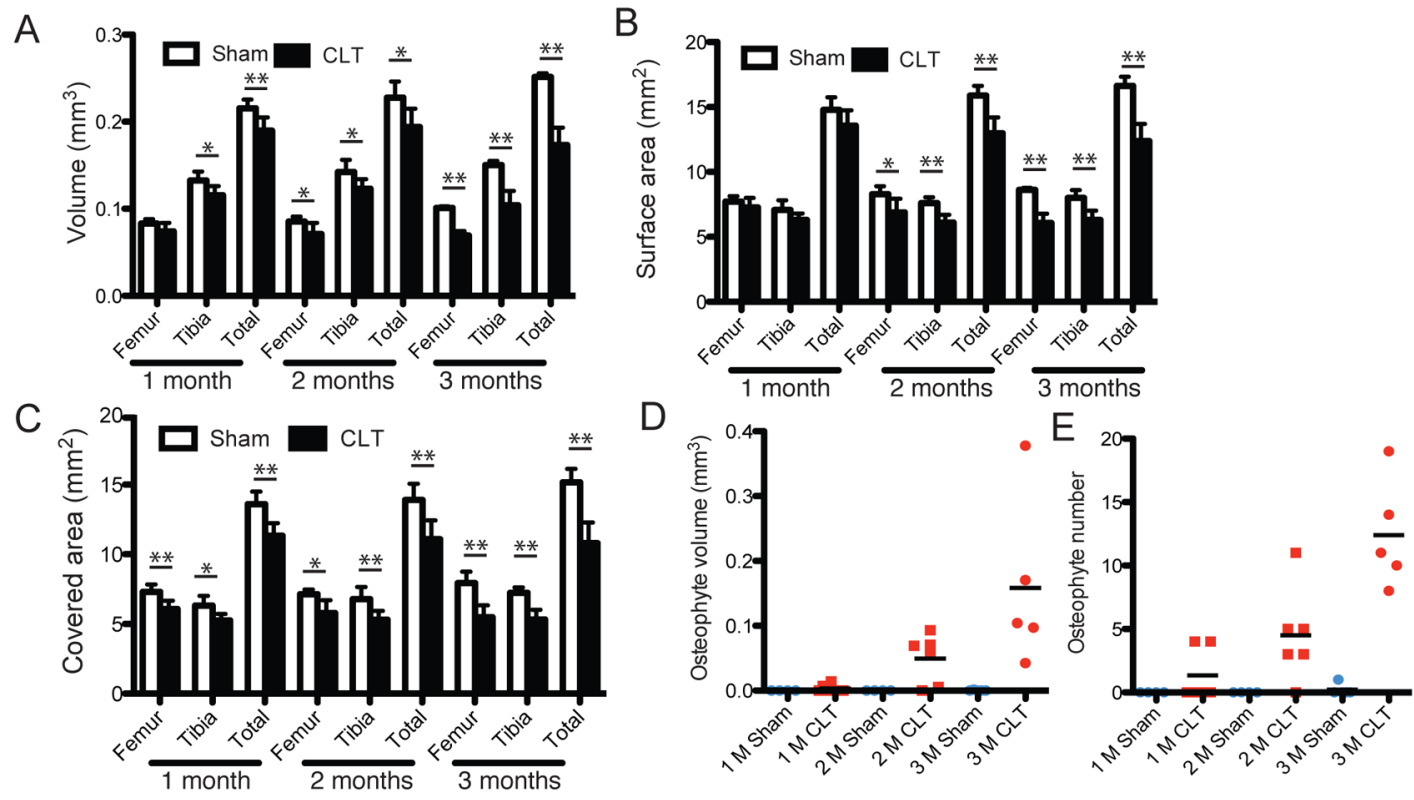

Figure 4. Quantification of cartilage loss and bone remodeling in sham and CLT joints (A) Volume of femur, tibia and total cartilage of sham transected and CLT joints at different time points after surgery. (B) Surface area of femur, tibia and total cartilage of sham transected and CLT joints at different time points after surgery. (C) Bone surface area covered by cartilage of femur, tibia and total of sham transected and CLT joints at different time points after surgery. (D) Osteophyte and heterotopic bone volume in knee joints at 1 month, 2 months and 3 months after sham transection and CLT. (E) Osteophyte and heterotopic bone number in knee joints at 1 month, 2 months and 3 months after sham transection and CLT. $\mathrm{N}=5$ in all the analysis. All error bars represent s.d. All the significant comparisons are labeled, $* \mathrm{p}<0.05, * * \mathrm{p}<0.01$. 
A

B

C
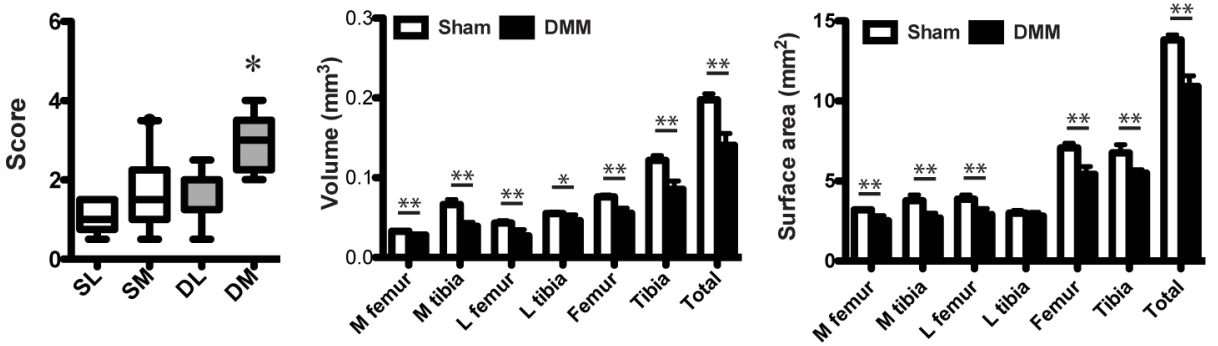

D

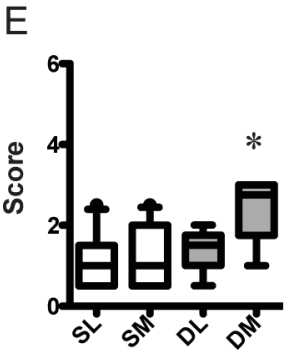

$\mathrm{F}$

$\mathrm{G}$
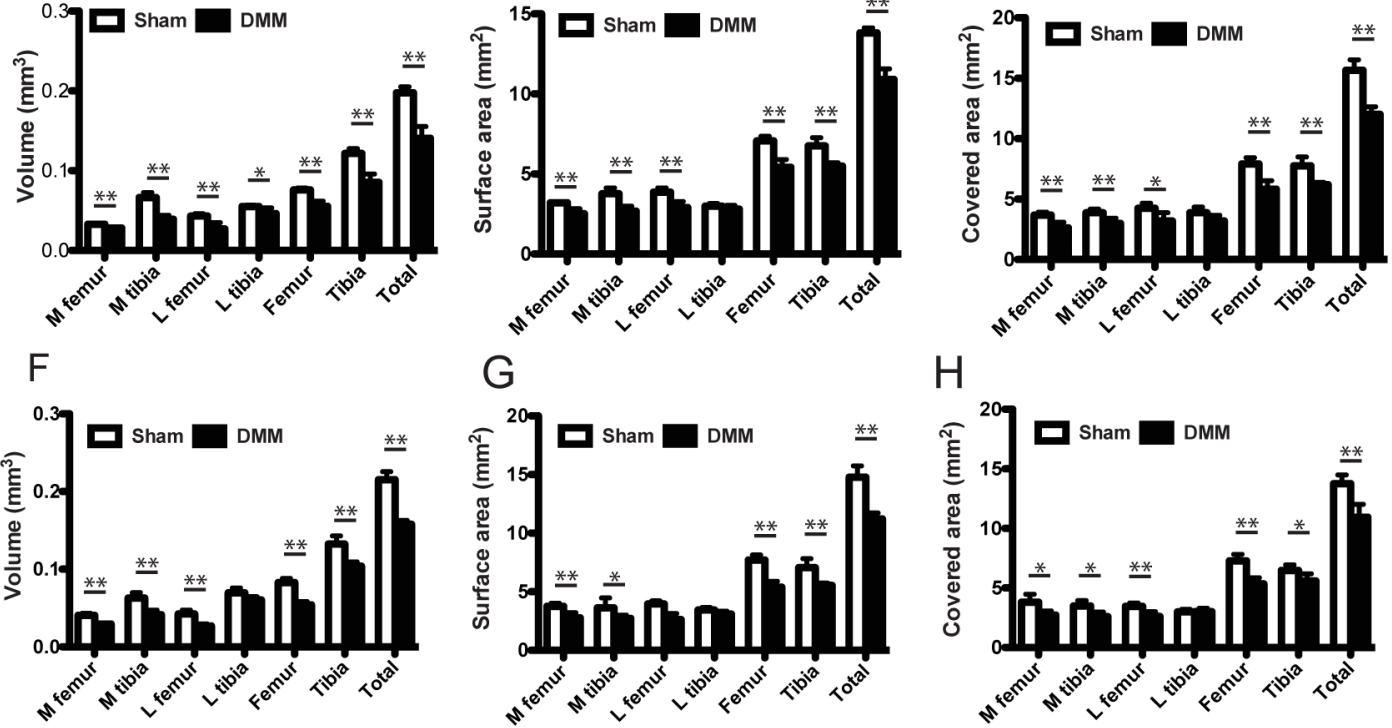

$\mathrm{H}$

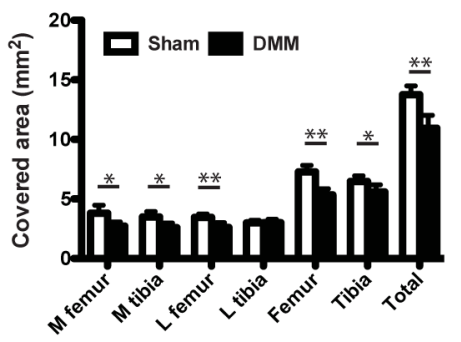

Figure 5. Quantification of cartilage loss in sham and DMM joints in 129SV/EV and FVB/N mice (A) Box plot of maximum score of DMM knee femoral articular cartilage compared to sham transection at 1 month after surgery. The group labeled with * is statistically significantly different from all other groups. SL: sham lateral, SM: sham medial, DL, DMM lateral, DM: DMM medial $(n=8$, box plot showing median and interquartile range, whiskers represent 10 to 90 percentile) (B-D) Volume (B), surface area (C) and bone surface area covered by cartilage (D) of medial femur (M femur), medial tibia (M tibia), lateral femur (L femur), lateral tibia (M tibia), femur, tibia and total cartilage of sham transected and DMM joint 1 month after surgery in $129 \mathrm{SV} / \mathrm{EV}$ mice. (E-F) Same as (A-D) but in FVB/N mice. N=5 in all the analysis. All error bars represent s.d. All the significant comparisons are labeled, $* \mathrm{p}<0.05, * * \mathrm{p}<0.01$. 
